\title{
Wideband Electromagnetic Energy Harvesting from a Rotating Wheel
}

\author{
Yu-Jen Wang, Sheng-Chih Shen and Chung-De Chen
}

Additional information is available at the end of the chapter

http://dx.doi.org/10.5772/50739

\section{Introduction}

Harvesting kinetic energy from the environment is an ideal alternative to traditional electrochemical batteries as a power source for low-power electronics such as pressure sensors and thermometers. A tire pressure monitoring system (TPMS) is a key device in preventing a flat tire, which is one of the most frequent causes of car accidents. The U.S.A., European Union, and a number of Asian countries are currently considering the implementation of TPMSrelated legislation. Existing TPMS are powered by a battery, but there are drawbacks to these batteries, such as their limited durability and environmental pollution upon disposal. For this reason, this chapter is aimed at developing a TPMS energy harvester in a rotating wheel.

Researches on energy harvesting from the environment for TPMS have grown in the recent years. The first part of this chapter introduces some of the representative ways to scavenge energy from a rotating wheel or tire. A wide bandwidth or similar frequency-adjusting energy harvesting device is necessary to power a TPMS. This is an emerging research topic with great challenge and the need for further improvement. This chapter presents a wellweighted pendulum with nonlinear effects to help the pendulum adjust its natural frequency to match the wheel rotation frequency. This chapter also formulates mathematical models of the weighted pendulum using the Euler-Lagrange formulation according to arbitrary configurations. This well-weighted pendulum oscillates at various wheel speeds with a larger swing angle and angular velocity than an ill-weighted pendulum design. Electromagnetic induction converts the kinetic energy produced by the pendulum into electrical energy. Numerical analysis reveals that a well-weighted pendulum generates hundreds of micro-Watts. This chapter establishes and analyzes dynamic models with electromechanical couplings, and introduces a novel circular Halbach array to augment magnetic strength on the coil side of the array. At an optimum external resistance, the power output of the well-weighted pendulum was approximately 300 to 550 micro-Watts at 
200-500 rpm. This performance demonstrated that this wideband electromagnetic energy harvester has the potential to replace traditional batteries in a TPMS.

\section{Energy harvester for a tire pressure monitor system}

A tire-pressure monitoring system (TPMS) is an electronic system designed to monitor the air pressure inside pneumatic tires on automobiles, airplanes, straddle-lift carriers, forklifts, and other vehicles (Fig. 1). A TPMS provides the driver with real-time tire pressure information and gives a warning message if the tire pressure falls below the recommended value. In 2000, the U.S. government issued the TREAD act, which forces car manufacturers to install TPMS in all new vehicles sold in the U.S. The European Union and a number of Asian countries are currently considering the implementation of TPMS-related legislation. The TPMS is expected to become one of the most popular electronic products in vehicle applications.

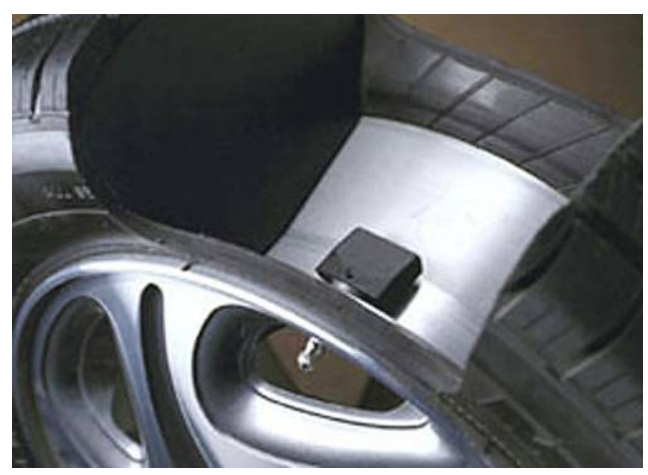

(a)

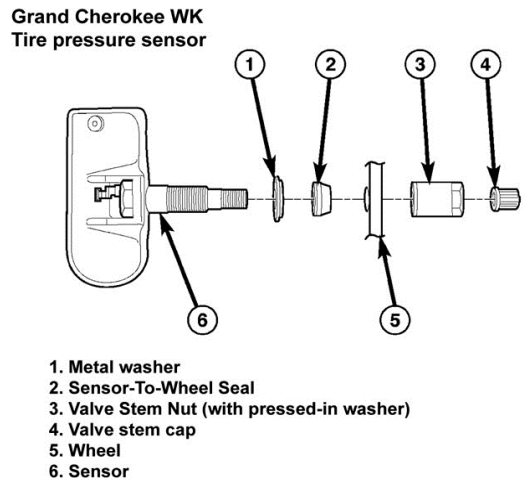

(b)

Figure 1. (a) TPMS module installed in a tire (b) Detailed components of TPMS by BMW.

A TPMS consists of a wireless pressure sensor inside each tire and a receiver in the car. Existing systems are powered by lithium batteries, but there are drawbacks to these batteries: limited durability, relatively large size and weight, and environmental pollution upon disposal. As a result, they have gradually become less favored by the public. For this reason, research on harvesting energy from the environment for TPMS has grown over the past few years (Hatipoglu \& Urey, 2009; Matsuzaki \& Todoroki, 2008). This chapter discusses a TPMS energy harvester device to satisfy the critical demand for a sustainable TPMS power supply.

\subsection{A review of previous research on wide-bandwidth energy-harvesting methods}

Instead of relying on embedded batteries, an energy harvesting device converts mechanical vibration energy into electricity. Possible methods of powering wireless devices installed on 
a vehicle include harvesting energy from a rotating tire or road vibrations. Previous reports regarding energy harvesting from a rotating wheel or tire (Hatipoglu \& Urey, 2009; Lohndorf et al., 2007; Matsuzaki \& Todoroki, 2008; Roundy 2008) have utilized vibration energy. One of the critical issues is that the maximal frequency band of the energy harvesting device is limited and cannot be chosen arbitrarily (Roundy 2008).

(Leland et al., 2006) presented a tuneable-resonance vibration energy scavenger that axially compresses a piezoelectric bimorph to decrease its resonance frequency. They showed that an axial preload can adjust the resonance frequency of a simply-supported bimorph to $24 \%$ below its unloaded resonance frequency. The power output to a resistive load was $65-90 \%$ of the nominal value at frequencies $19-24 \%$ below the unloaded resonance frequency. Prototypes produced $300-400 \mu \mathrm{W}$ of power at driving frequencies of $200-250 \mathrm{~Hz}$. However, the compressive axial preload of the piezoelectric device cannot automatically adjust itself. The other method of ensuring that the energy harvester has a self-tuning resonance frequency capability to match the base motion frequency is integrating the harvester with an accelerometer, a displacement sensor, and an actuator to tune the stiffness of the harvester based on the base vibration frequency (Leland \& Wright, 2006). Passive frequency-adjusting energy-harvesting mechanisms have grown in use over the past few years (Daminakis et al., 2005; Gu \& Livermore, 2010; Lee et al., 2007; Mansour et al., 2010; Marzencki et al., 2009). (Youngsman et al., 2005) presented a model for a frequency-adjustable vibration energy harvester.

(Sari et al., 2008) developed a wideband electromagnetic vibration-to-electrical micro power generator. This generator covers a wide band of external vibration frequencies by implementing a number of serially connected cantilevers of different lengths and varying natural frequencies. This device generated $0.4 \mu \mathrm{W}$ of continuous power with $10 \mathrm{mV}$ voltage at an external vibration frequency range of $4.2-5 \mathrm{kHz}$, covering a band of $800 \mathrm{~Hz}$. Possible disadvantages of this approach are that the maximum power is too small to power a TPMS, and the suitable vibration frequency is too high for a rotating wheel. Other researchers have attempted to develop wide-band structures to achieve high-efficiency energy-scavenging devices (Marinkovic \& Koser, 2009; Zhang \& Chen, 2010).

(Stanton et al., 2009) validated a nonlinear energy harvester capable of bidirectional hysteresis. This design invokes hardening and softening responses within the quadratic potential field of a power generating piezoelectric beam (with a permanent magnet end mass) by tuning nonlinear magnetic interactions. This technique increases the bandwidth of the device from $12 \pm 0.5 \mathrm{~Hz}$ to $11 \sim 14 \mathrm{~Hz}$. To consider the nonlinear effects in electromagnetic harvest mechanisms, Dallago et al., 2010 presented an analytical model that considers the non-linear electromagnetic repulsion force and the flux linkage of the coil.

Bower \& Arnold, 2009 developed a non-resonant energy harvester that employs a spherical magnet ball that moves arbitrarily in a cavity wrapped with copper coil windings. This harvester demonstrated root-mean-square voltages ranging from 80 to $700 \mathrm{mV}$. Platt et al., 2005, developed a low-frequency electric power generator using PZT ceramics for the same purpose. 
A wide bandwidth or similar frequency-adjusting energy harvesting device is necessary for a TPMS. This is an emerging research topic with great challenges and the need for further improvement. This chapter proposes the use of a novel well-weighted pendulum method to harvest kinetic energy from a rotating wheel. In this design, the pendulum oscillates in response to periodic changes in the tangential component of gravity. The greatest advantage of this approach is that the rotating frequency of the wheel increases linearly as the car speeds up. Unlike traditional energy-harvesting devices, which have a fixed natural frequency, the radial acceleration of the rotating wheel will raise the pendulum's natural frequency of oscillation as the car increases its speed.

\subsection{TPMS power consumption}

Knowing the power consumption of TPMS is a prerequisite to designing a suitable energy harvester. Fig. 2 shows the all-in-one package block diagram of the TPMS produced by Orange Electronics, which includes a pressure sensor, an 8-bit microcontroller (MCU), an RF transmitter, and a 2-axis accelerometer sensor. This TPMS is installed on the wheel rims to provide independent, real-time air pressure measurements for each tire that can be transmitted to the vehicle instrument cluster to instantly inform the driver.

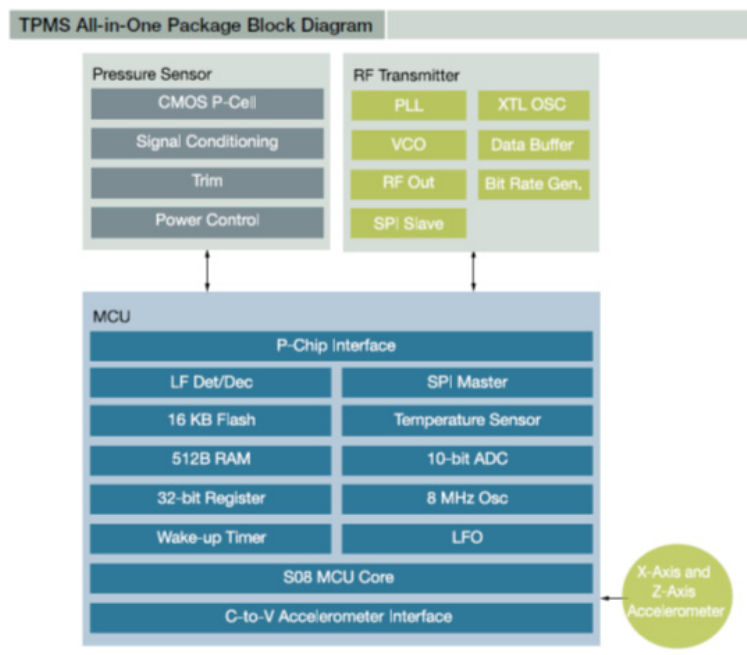

Figure 2. TPMS block diagram by Orange Electronics.

Fig. 3 depicts the Orange Electronics P409S TPMS, P409S, employed in the experiment. The low-current measurement circuit is in-series connected with the TPMS (Fig. 4). From LF411, the output voltage $V_{o}$ provides $2 \mathrm{~mA} / \mathrm{V}$ exchange rate. Table 1 lists the operation modes and power consumption of the TPMS. The whole operation, which consists of pressure measurement, RF transmitter activation, temperature measurement, and idle mode, lasts 130.2 seconds and consumes average power $48.1 \mu \mathrm{w}$. 
Pressure sensor

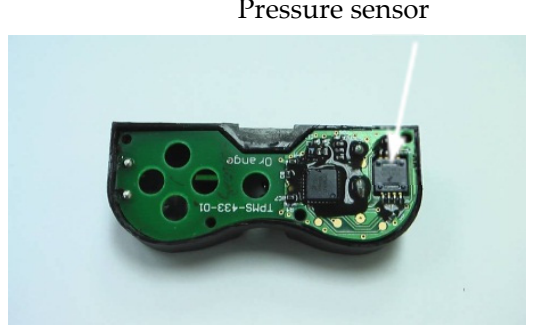

Battery

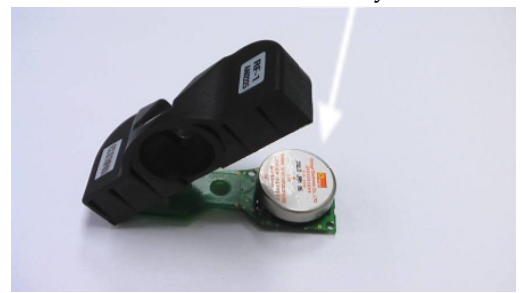

Figure 3. Orange Electronics P409S TPMS.

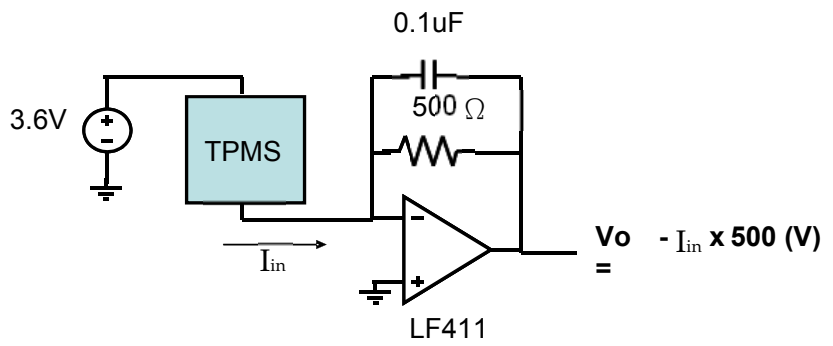

Figure 4. Low current measurement circuit.

\begin{tabular}{|c|c|c|c||c||c|c|}
\hline \hline Item & Action & $\begin{array}{c}\text { Number } \\
\text { of times }\end{array}$ & $\begin{array}{c}\text { Duration } \\
(\mathrm{sec})\end{array}$ & $\begin{array}{c}\text { Peak } \\
\text { current } \\
(\mathrm{mA})\end{array}$ & $\begin{array}{c}\text { Average } \\
\text { current } \\
(\mathrm{mA})\end{array}$ & $\begin{array}{c}\text { Average } \\
\text { power } \\
(\mathrm{mW})\end{array}$ \\
\hline A & Pressure sensor active & 36 & 0.01 & 2.56 & 1.20 & 4.32 \\
\hline B & Idle mode & 42 & 3.1 & 0.00260 & 0.00260 & 0.00936 \\
\hline C & RF signal & 5 & 0.042 & 4.75 & 4.33 & 15.6 \\
\hline D & $\begin{array}{c}\text { Pressure and Temp. } \\
\text { measurement }\end{array}$ & 1 & 0.01 & 2.56 & 1.75 & 6.30 \\
\hline
\end{tabular}

Table 1. Operation mode and power consumption of TPMS

\section{Kinetic energy of the wheel}

The pressure sensing module of TPMS was mounted under a rubber tire to measure the tire pressure. The first step in designing the energy harvesting device for TPMS was to determine the kinetic energy of a wheel. A 3-axis accelerometer and a radio frequency (RF) transmission module with a sampling rate $50 \mathrm{~Hz}$ were mounted on a wheel rim to collect kinematic data when the car was driving at any constant speed Fig. 5. The acceleration data were then transferred to a receiver connected to a laptop computer in the test vehicle. 


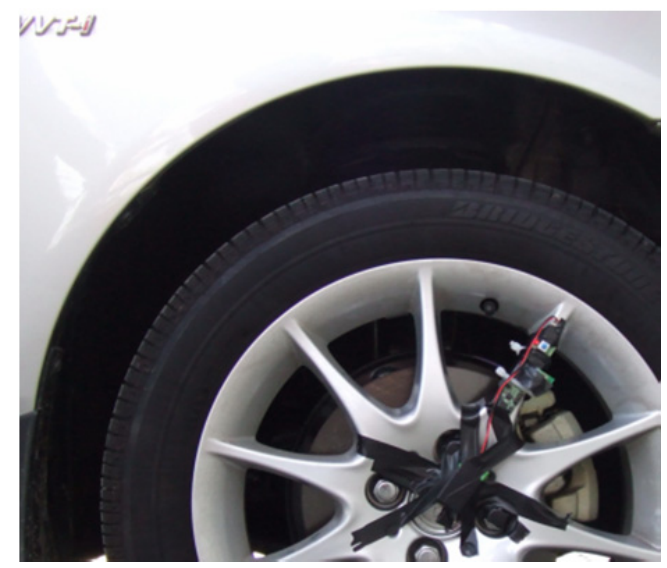

Figure 5. 3-axis accelerometer with the RF module mounted on the wheel.

Fig. 6 plots the normal and tangential accelerations of the accelerometer in red and blue lines, respectively, when the car travelled at a constant speed of $70 \mathrm{~km} / \mathrm{hr}(619 \mathrm{rpm})$ on an asphalt road. The normal acceleration resulted from both centripetal and gravitational forces during wheel rotation. Even at low speeds, such as $20 \mathrm{~km} / \mathrm{hr}$ (177 rpm), the centripetal force was 7 times larger than gravity. The tangential acceleration experienced only the tangential component of gravity. Fig. 7 shows the energy spectrum of the tangential acceleration, the peak of which is located at approximately $10 \mathrm{~Hz}$ and is very close to the wheel rotation frequency. This result was similar even when the test car was driving on a macadam road at various speeds. This indicates that other acceleration terms, such as that caused by tire vibration from the road, are insignificant. Therefore, the tangential acceleration at each point of the rim caused by the variability of the tangential component of gravity during rotation is a reliable energy source for a rotating wheel under any road conditions.

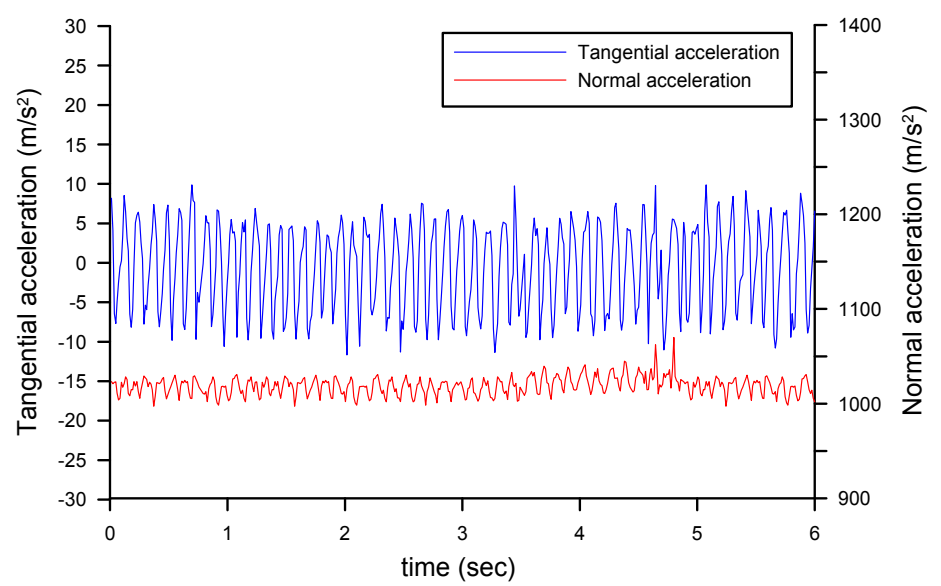

Figure 6. Normal and tangential acceleration data at $70 \mathrm{~km} / \mathrm{hr}(619 \mathrm{rpm})$. 


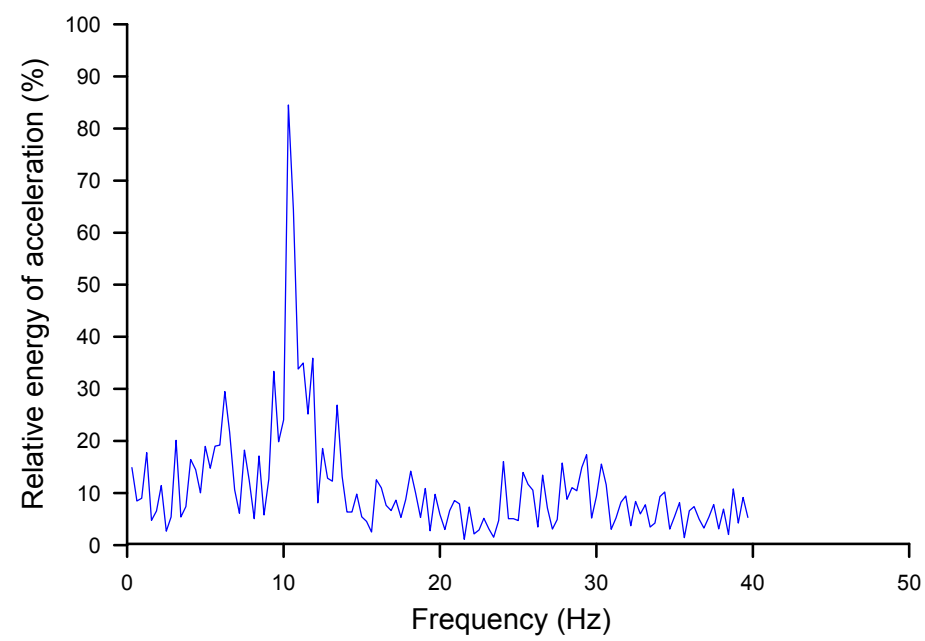

Figure 7. Energy spectrum of tangential acceleration at $70 \mathrm{~km} / \mathrm{hr}$.

\section{Weighted pendulum by arbitrary configurations}

This section derives the governing equation of the weighted pendulum from the arbitrary configuration to show the generality (Wang et al., 2012a). Consider a weighted pendulum mounted on a wheel (Fig. 8). The locations of the center and the pivot with reference to a fixed frame $(\mathrm{x}, \mathrm{y})$ are $\left(\mathrm{x}_{0}, 0\right)$ and $\left(\mathrm{x}_{1}, \mathrm{y}_{1}\right)$, respectively. Introducing the wheel rotation angle $\Theta$ in Fig. 8 gives

$$
\begin{aligned}
& x_{1}=x_{0}+R_{2} \sin \Theta \\
& y_{1}=R_{2} \cos \Theta
\end{aligned}
$$

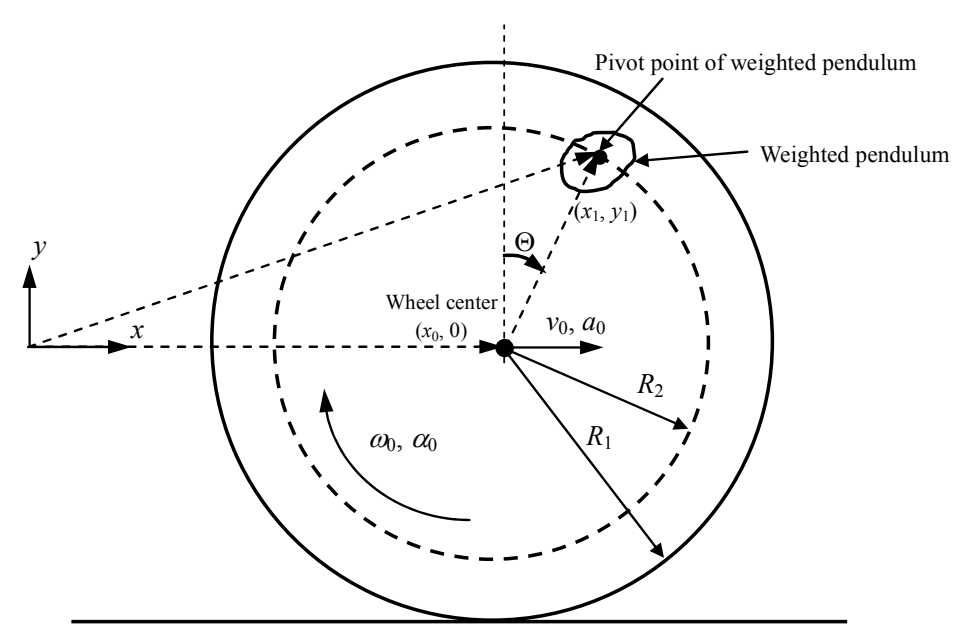

Figure 8. Schematic diagram of a rolling wheel. 
Fig. 9 shows the detailed configuration of the weighted pendulum, which can be an arbitrary shape but whose rotation axis is not located at the center of mass. This study defines a local coordinate $(\bar{x}, \bar{y})$ attached to the weighted pendulum such that its origin is located at the pivot and its center of mass is located at the extended line of the $\bar{y}$-axis. Considering an infinitesimal element $d m$ on the weighted pendulum, the position of $d m$ can be defined by a distance $r$ and an angle $\phi$ (Fig. 9). $\theta$ is the swing angle of the weighted pendulum. Note that the profile of the weighted pendulum represented by the dashed line in Fig. 9 denotes an arbitrary swing angle $\theta$ with reference to the pivot point while the solid line denotes a specific position $\theta=0$. The swing angle can be considered as the relative position between the weighted pendulum and the wheel. Further define an angle $\varphi$ such that

$$
\phi+\theta+\varphi-\Theta=\frac{\pi}{2}
$$

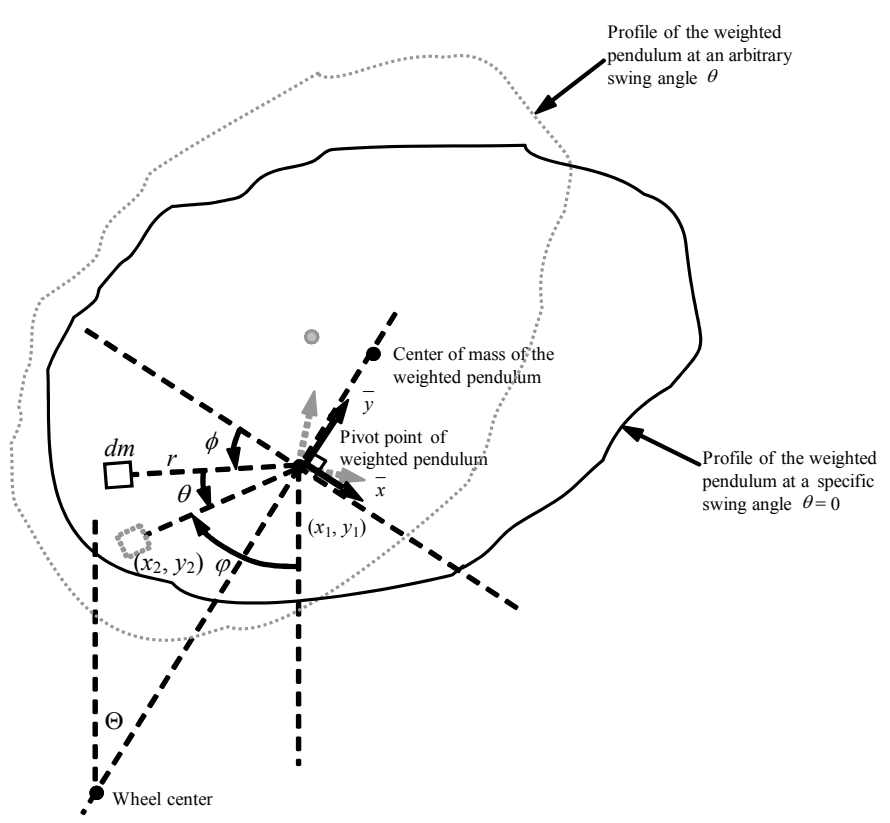

Figure 9. Diagram of a weighted pendulum.

For an arbitrary swing angle $\theta$, the location of $d m$ with respect to $(\mathrm{x}, \mathrm{y})$ is

$$
\begin{aligned}
& x_{2}=x_{0}+R_{2} \sin \Theta-r \sin \varphi \\
& y_{2}=R_{2} \cos \Theta-r \cos \varphi
\end{aligned}
$$

where $R_{2}$ is the distance between the pivot and the wheel center. By differentiating Eq. (3) with respect to time $\mathrm{t}$, the velocity of $d m$ can be obtained as follows: 


$$
\begin{aligned}
& \dot{x}_{2}=v_{0}+R_{2} \dot{\Theta} \cos \Theta-r \dot{\varphi} \cos \varphi \\
& \dot{y}_{2}=-R_{2} \dot{\Theta} \sin \Theta+r \dot{\varphi} \sin \varphi
\end{aligned}
$$

where $\mathrm{v}_{0}$ is the speed of the wheel center and $\dot{\Theta}$ is the wheel rotation speed. The equations of motion of the weighted pendulum can be derived using the Lagrange equation. Based on Eq. (4), the kinetic energy of the weighted pendulum can be obtained by integrating over the volume $\bar{V}$ with respect to the local coordinate $(\bar{x}, \bar{y})$ :

$$
\begin{aligned}
T= & \frac{1}{2} \int\left(\dot{x}_{2}^{2}+\dot{y}_{2}^{2}\right) d m \\
& =\frac{1}{2} \int\left[v_{0}^{2}+R_{2}^{2} \dot{\Theta}^{2}+r^{2} \dot{\varphi}^{2}+2 v_{0}\left(R_{2} \dot{\Theta} \cos \Theta-r \dot{\varphi} \cos \varphi\right)\right. \\
& \left.-2 R_{2} r \dot{\Theta} \dot{\varphi} \cos (\Theta-\varphi)\right] \rho d \bar{V}
\end{aligned}
$$

Eq. (5) uses the relation

$$
d m=\rho d \bar{V}
$$

where $\varrho$ is density of the weighted pendulum and $d \bar{V}$ is an infinitesimal volume. The potential energy is

$$
V=g \int\left[R_{2} \cos \Theta-r \cos \varphi\right] \rho d \bar{V}
$$

where $\mathrm{g}$ is the gravitational acceleration. According to the Lagrangian function L:

$$
\begin{aligned}
L= & T-V \\
& =\frac{1}{2} \int\left[v_{0}^{2}+R_{2}^{2} \dot{\Theta}^{2}+r^{2} \dot{\varphi}^{2}+2 v_{0}\left(R_{2} \dot{\Theta} \cos \Theta-r \dot{\varphi} \cos \varphi\right)-2 R_{2} r \dot{\Theta} \dot{\varphi} \cos (\Theta-\varphi)\right. \\
& \left.-2 g\left(R_{2} \cos \Theta-r \cos \varphi\right)\right] \rho d \bar{V}
\end{aligned}
$$

According to the Euler-Lagrange formulation, the equation of motion can be written as:

$$
\frac{d}{d t}\left(\frac{\partial L}{\partial \dot{\varphi}}\right)-\frac{\partial L}{\partial \varphi}=0
$$

Substituting Eq. (8) into Eq. (9) yields the following equation:

$$
\begin{array}{r}
\int \rho r^{2} \ddot{\varphi} d \bar{V}-a_{0} \int \rho r \cos \varphi d \bar{V}-R_{2} \ddot{\Theta} \int \rho r \cos (\Theta-\varphi) d \bar{V} \\
+R_{2} \dot{\Theta}^{2} \int \rho r \sin (\Theta-\varphi) d \bar{V}+g \int \rho r \sin \varphi d \bar{V}=0
\end{array}
$$

in which $a_{0}=\ddot{x}_{0}$. Substituting Eq. (9) into Eq. (10) yields

$$
\begin{aligned}
& \left(\int \rho r^{2} d \bar{V}\right)(\ddot{\Theta}-\ddot{\theta})-a_{0} \int \rho r \sin (\phi+\theta-\Theta) d \bar{V} \\
& \quad-R_{2} \ddot{\Theta} \int \rho r \sin (\phi+\theta) d \bar{V}-R_{2} \dot{\Theta}^{2} \int \rho r \cos (\phi+\theta) d \bar{V}+g \int \rho r \cos (\phi+\theta-\Theta) d \bar{V}=0
\end{aligned}
$$


Recall that the $\bar{y}$-axis passes through the center of mass of the weighted pendulum. Therefore, the mass distribution is symmetric with respect to the $\bar{y}-\bar{z}$ plane, which gives $\int \rho r \cos \phi d \bar{V}=0$. Eq. (11) then becomes

$$
\begin{aligned}
& \left(\int \rho r^{2} d \bar{V}\right) \ddot{\theta}-R_{2} \dot{\Theta}^{2}\left(\int \rho r \sin \phi d \bar{V}\right) \sin \theta \\
& =\left(\int \rho r^{2} d \bar{V}\right) \ddot{\Theta}-a_{0}\left(\int \rho r \sin \phi d \bar{V}\right) \cos (\theta-\Theta)- \\
& R_{2} \ddot{\Theta}\left(\int \rho r \sin \phi d \bar{V}\right) \cos \theta-g\left(\int \rho r \sin \phi d \bar{V}\right) \sin (\theta-\Theta)
\end{aligned}
$$

where $\int \rho r^{2} d \bar{V}=I$ is the mass moment of inertia. At this point, and without loss of generality, assume a constant wheel speed and a small swing angle of the pendulum to facilitate the study of the natural frequency of the weighted pendulum. After linearization, the equation of motion can be written as

$$
\left(\int \rho r^{2} d \bar{V}\right) \ddot{\theta}-R_{2} \dot{\Theta}^{2}\left(\int \rho r \sin \phi d \bar{V}\right) \theta=-g\left(\int \rho r \sin \phi d \bar{V}\right) \sin \Theta
$$

The natural frequency $\omega_{\mathrm{n}}$ of the weighted pendulum is

$$
\omega_{n}=\left(-R_{2} \dot{\Theta}^{2} \frac{\int \rho r \sin \phi d \bar{V}}{\int \rho r^{2} d \bar{V}}\right)^{1 / 2}
$$

This study defines the characteristic length, $L^{*}$, as

$$
L^{*}=-\frac{\int \rho r^{2} d \bar{V}}{\int \rho r \sin \phi d \bar{V}}
$$

Because of the definition of the local coordinate $(\bar{x}, \bar{y})$, sin $\phi$ is a negative number. Therefore, the characteristic length $L^{*}$ is a positive number. After linearization, the natural frequency at a constant wheel speed can be written as

$$
\omega_{n}=\dot{\Theta} \sqrt{\frac{R_{2}}{L^{*}}}
$$

Note that $\omega_{n}$ is proportional to the wheel rotation frequency $\dot{\Theta}$. If the weighted pendulum is designed to make

$$
L^{*}=R_{2}
$$

then the natural frequency of the weighted pendulum is equal to the wheel rotation frequency at any car speed to achieve high-efficiency energy harvesting. By integrating the weighted pendulum with magnets and coils, kinetic energy can be transformed into electrical energy during wheel rotation. 
By adding the damping term, Eq. (12) can be written as

$$
\begin{aligned}
& \left(\int \rho r^{2} d \bar{V}\right) \ddot{\theta}+C_{T} \dot{\theta}-R_{2} \dot{\Theta}^{2}\left(\int \rho r \sin \phi d \bar{V}\right) \sin \theta \\
& =\left(\int \rho r^{2} d \bar{V}\right) \ddot{\Theta}-a_{0}\left(\int \rho r \sin \phi d \bar{V}\right) \cos (\theta-\Theta)- \\
& R_{2} \ddot{\Theta}\left(\int \rho r \sin \phi d \bar{V}\right) \cos \theta-g\left(\int \rho r \sin \phi d \bar{V}\right) \sin (\theta-\Theta)
\end{aligned}
$$

where $C_{T}^{*}$ is the total generalized damping constant. Eq. (18) can then be rewritten as

$$
\ddot{\theta}+C_{T}^{*} \dot{\theta}-\frac{R_{2} \dot{\Theta}^{2}}{L^{*}} \sin \theta=\ddot{\Theta}-\frac{a_{0}}{L^{*}} \cos (\theta-\Theta)-\frac{R_{2} \ddot{\Theta}}{L^{*}} \cos \theta-\frac{g}{L^{*}} \sin (\theta-\Theta)
$$

in which $C_{T}^{*}=C_{e}^{*}+C_{m}^{*}$ and $C_{T}^{*}=\frac{C_{T}}{\left(\int \rho r^{2} d \bar{V}\right)} \cdot C_{e}^{*}$ and $C_{m}^{*}$ are the generalized electromagnetic damping and the generalized mechanical damping caused by the electromagnetic force and the friction of motion, respectively. By assuming a constant car speed, Eq. (19) becomes

$$
\ddot{\theta}+C_{T}^{*} \dot{\theta}-\frac{R_{2} \dot{\Theta}^{2}}{L^{*}} \sin \theta=-\frac{g}{L^{*}} \sin (\theta-\Theta)
$$

This section discusses the dynamic behavior of the weighted pendulum by solving the Eq. (19) using Runge-Kutta method. Eq. (19) shows that the dynamic behavior can be determined by two independent parameters: the characteristic length $L^{*}$ and the total generalized damping constant $C_{T}^{*}$, if the dimensions of the wheel $\left(R_{1}\right.$ and $\left.R_{2}\right)$, the velocity and acceleration of the car are known. The following parameters are fixed in all of the following numerical studies: $R_{1}=0.300 \mathrm{~m}, R_{2}=0.203 \mathrm{~m}, \mathrm{~g}=9.81 \mathrm{~m} / \mathrm{s}^{2}, \mathrm{I}=1.847 \times 10^{-6} \mathrm{~kg}-\mathrm{m}^{2}$. I is the mass moment of inertia of the rotor. As an illustrative example, the characteristic length and generalized damping constant are $0.192 \mathrm{~m}$ and $0.70 \mathrm{~N}-\mathrm{s} / \mathrm{kg} / \mathrm{m}$, respectively. Fig. 10 shows the swing angle $\theta$ driven by gravity in the time domain when the wheel speed is 350 rpm with the initial conditions $\theta(0)=\pi / 3$ and $\dot{\theta}(0)=0$. To simplify the description in the following figures and discussions, the maximum swing angle, $\theta_{\max }$, is defined by the largest swing angle value in the positive direction.

Fig. 11 shows the maximum swing angle at the steady state, $\theta_{\max }$, at a wheel speed of 350 rpm and the initial conditions $\theta=0$ and $\dot{\theta}=0$ under various damping constants. The swing angle strongly depends on $L^{*}$ when damping is small. For large damping, the swing angle becomes small and the dynamic system becomes linear. The validity of the numerical results of swing angle for large damping was confirmed by the linear vibration theory. Fig. 11 shows that small damping yields a large swing angle. However, a large swing angle does not guarantee a large amount of power converted.

Fig. 12 shows the steady-state average power for a wheel speed $350 \mathrm{rpm}$ and initial conditions $\theta=0$ and $\dot{\theta}=0$ under various damping constants. This figure reveals a 
compromise between swing angle and damping constant. The optimum total generalized damping to obtain a large amount of power converted is approximately $1.6 \mathrm{~N}-\mathrm{s} / \mathrm{kg} / \mathrm{m}$ to 3.0 $\mathrm{N}-\mathrm{s} / \mathrm{kg} / \mathrm{m}$. Fig. 11 and Fig. 12 indicate that the best $L^{*}$ ranges from $0.190 \mathrm{~m}$ to $0.203 \mathrm{~m}$ for a specific damping constant.

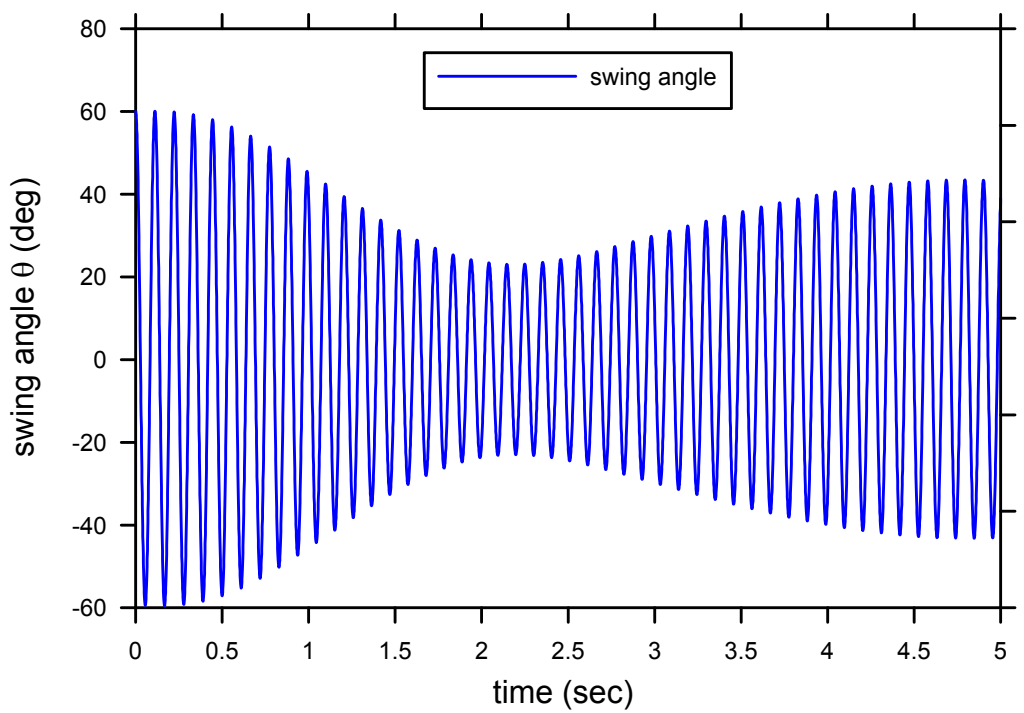

Figure 10. Transient response of the swing angle at the wheel speed $350 \mathrm{rpm}$ and initial conditions $\theta(0)=\pi / 3$ and $\dot{\theta}(0)=0$.

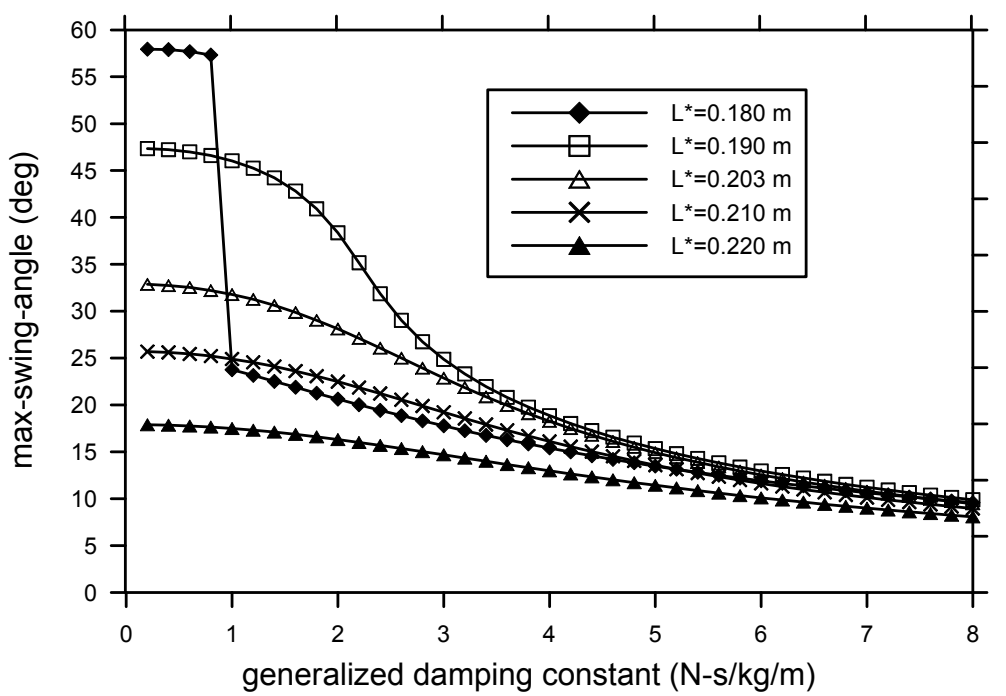

Figure 11. Variation of the steady-state swing angle for the wheel speed $350 \mathrm{rpm}$ and the initial conditions $\theta=0$ and $\dot{\theta}=0$ under various total generalized damping constants. 
The swing motion of a weighted pendulum driven by gravity exhibits nonlinear behavior. Eq. (19) describes the dynamic behavior of the weighted pendulum, and the requirements of Eq. (17) cause the system to oscillate at resonance at any wheel speed. Fig. 13 shows the variation of maximum swing angle at the steady state under various $L^{*}$ and wheel rotation speeds with total generalized damping constant $\mathrm{CT}^{*}=0.70 \mathrm{~N}-\mathrm{s} / \mathrm{kg} / \mathrm{m}$. When $L^{*}=0.203 \mathrm{~m}$ and the condition $L^{*}=R_{2}$ is met, the swing angle varies continuously at different wheel speeds. Compared with with other situations, the maximum swing angle when $L^{*}>R_{2}$ is smaller than that when $L^{*}=R_{2}$. This means that the power generated decreases when $L^{*}$ exceeds $R_{2}$. When $L^{*}<R_{2}$, the maximum swing angle exhibits a sudden discontinuous jump above the critical wheel rotation speed. Using the case $L^{*}=0.190 \mathrm{~m}$ as an example, the amplitude gradually decreases with an increasing frequency of wheel rotation until point $a$ is reached, and then suddenly jumps to a smaller value with an increasingly small rotation frequency, as indicated by point $b$. The narrow region between point $a$ and point $b$ in Fig. 13 is unstable. Beyond point $b$, the amplitude of the maximum swing angle keeps continuously decreasing. The results above indicate that when $L^{*}$ is exactly equal to $R_{2}$, a steady output power is provided at any speed. This provides evidence of the advantage of using a well-weighted pendulum.

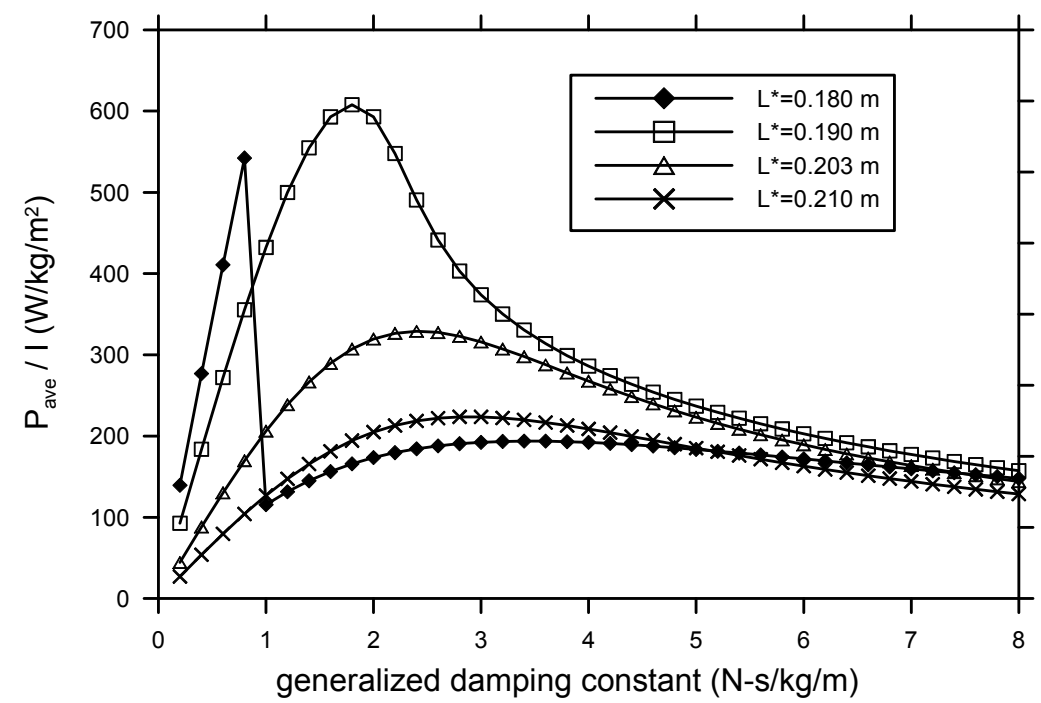

Figure 12. Variation of the steady-state average power for the wheel speed $350 \mathrm{rpm}$ and the initial conditions $\theta=0$ and $\dot{\theta}=0$ under various total generalized damping constants.

The discussion above assumes that the car velocity is constant. The following discussion considers two non-constant car velocity modes, acceleration and deceleration (Fig. 14). The characteristic length and total generalized damping constant are $0.203 \mathrm{~m}$ and $0.70 \mathrm{~N}-\mathrm{s} / \mathrm{kg} / \mathrm{m}$, respectively, and the initial conditions are $\theta(0)=0$ and $\dot{\theta}(0)=0$. In the first 10 seconds, the constant speed mode causes the steady-state value of the maximum angular velocity to 
reach approximately $17 \mathrm{rad} / \mathrm{s}$ (Fig. 14 (a)). In the time interval from 10 to 25 seconds, the car accelerates at a rate of $1.48 \mathrm{~m} / \mathrm{s}^{2}$, during which the angular velocity increases at a rate of approximately $0.87 \mathrm{rad} / \mathrm{s}^{2}$, and the high wheel speed increases the maximum power to approximately $800 \mathrm{~W} / \mathrm{Kg} / \mathrm{m}^{2}$. In the time interval from 25 to 35 seconds, the variation of maximum power and maximum angular velocity is small because the car speed remains constant. In the period from 35 to 45 seconds, the car decelerates at $2.22 \mathrm{~m} / \mathrm{s}^{2}$, causing the maximum angular velocity to drop at a rate of approximately $0.95 \mathrm{rad} / \mathrm{s}^{2}$ and the maximum power to drop at a rate of $43 \mathrm{~W} / \mathrm{Kg} / \mathrm{m}^{2}$ per second. Fig. 14 (b) shows that the maximum power and maximum angular velocity increases linearly with constant acceleration or deceleration. When the well-weighted pendulum satisfies the requirement of Eq. (17), the variation in maximum power and maximum angular velocity is continuous, and no jump phenomenon is apparent.

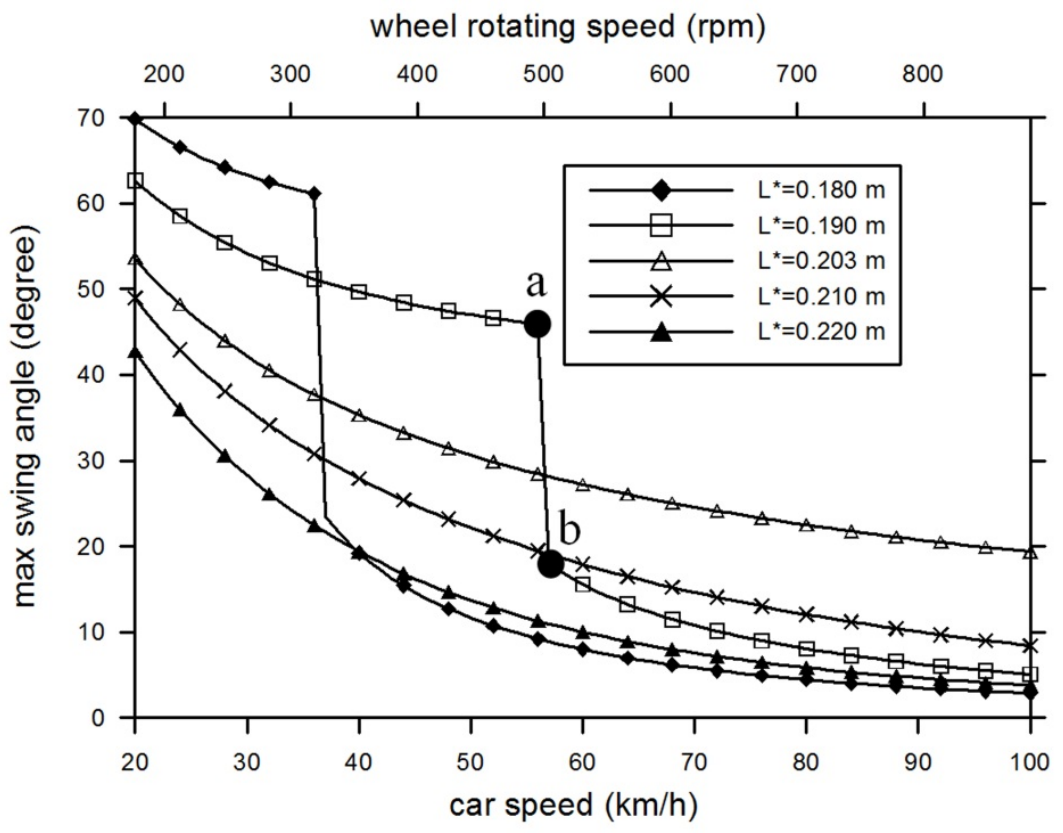

Figure 13. Variation of the steady-state swing angle under various $L^{*}$ and wheel speeds with initial conditions $\theta=0, \dot{\theta}=0$ and $C_{T}^{*}=0.70 \mathrm{~N}-\mathrm{s} / \mathrm{kg} / \mathrm{m}$.

The transient response analysis of the weighted pendulum after large car acceleration is also necessary. To give an example, the total generalized damping constant is $0.5 \mathrm{~N}-\mathrm{s} / \mathrm{Kg} / \mathrm{m}$ and the initial conditions are $\theta(0)=0$ and $\theta(0)=0$. In the first 10 seconds, the wheel rotation speed is constant at $200 \mathrm{rpm}$, which equals $22.6 \mathrm{~km} / \mathrm{hr}$ for $R_{1}=0.300 \mathrm{~m}$. In the time interval of acceleration from 10 to 20 seconds, the wheel speeds up with an angular acceleration $\alpha$ $=73.7 \mathrm{rad} / \mathrm{s}^{2}$ to reach a constant rotation speed of $710 \mathrm{rpm}$. This is equal to a $80.2 \mathrm{~km} / \mathrm{hr}$ car speed, and Fig. 15 shows the transient response of the weighted pendulum. The 
maximum swing angles of $L^{*}$ from 0.180 to 0.203 after $\alpha=73.7 \mathrm{rad} / \mathrm{s}^{2}$ are very close to the results of a small $\alpha=0.105 \mathrm{rad} / \mathrm{s}^{2}$. The maximum swing angles for $L^{*}=0.180$ and $0.190 \mathrm{~m}$ after $\alpha=148 \mathrm{rad} / \mathrm{s}^{2}$ are far smaller than after $\alpha=73.7 \mathrm{rad} / \mathrm{s}^{2}$ at the same final constant speed of $710 \mathrm{rpm}$ (Fig. 16). These results show that a huge angular acceleration can cause the jump phenomenon to occur for a critical value of $L^{*}$. Fig. 16 shows that the wellweighted pendulum of $L^{*}=R_{2}=0.203 \mathrm{~m}$ will not encounter the jump phenomenon when the angular acceleration speed is $148 \mathrm{rad} / \mathrm{s}^{2}$. The acceleration mode is equal to a car accelerating from 22.6 to $80.2 \mathrm{~km} / \mathrm{hr}$ in 5 seconds, which is the acceleration limit of a normal car on a highway.

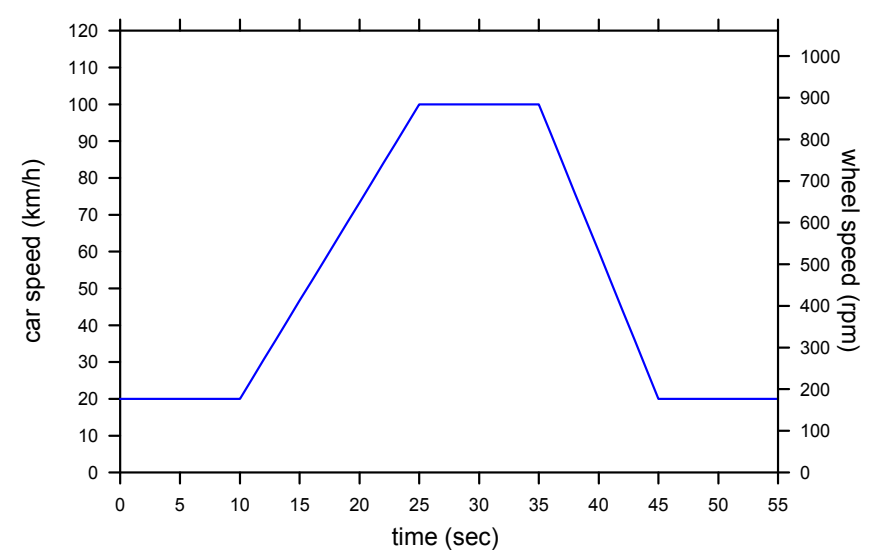

(a) Car speed mode

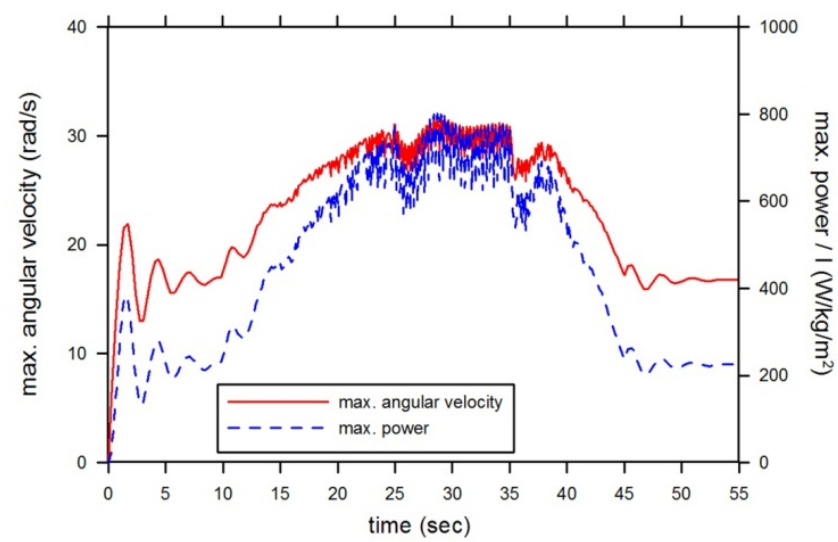

(b) Maximum power and angular velocity, $\dot{\theta}_{\max }$, under different car speeds.

Figure 14. Time domain responses of the well-weighted pendulum under car acceleration and deceleration. Characteristic length and total generalized damping constant are $0.203 \mathrm{~m}$ and $0.70 \mathrm{~N}$ $\mathrm{s} / \mathrm{kg} / \mathrm{m}$, respectively. 


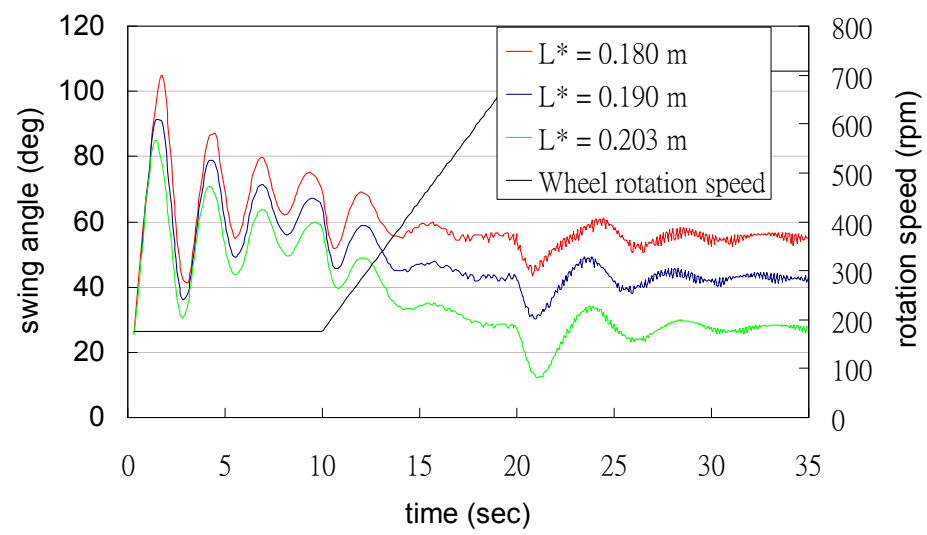

Figure 15. Maximum swing angle under various $L^{*}$ after angular acceleration of $73.7 \mathrm{rad} / \mathrm{s}^{2}$.

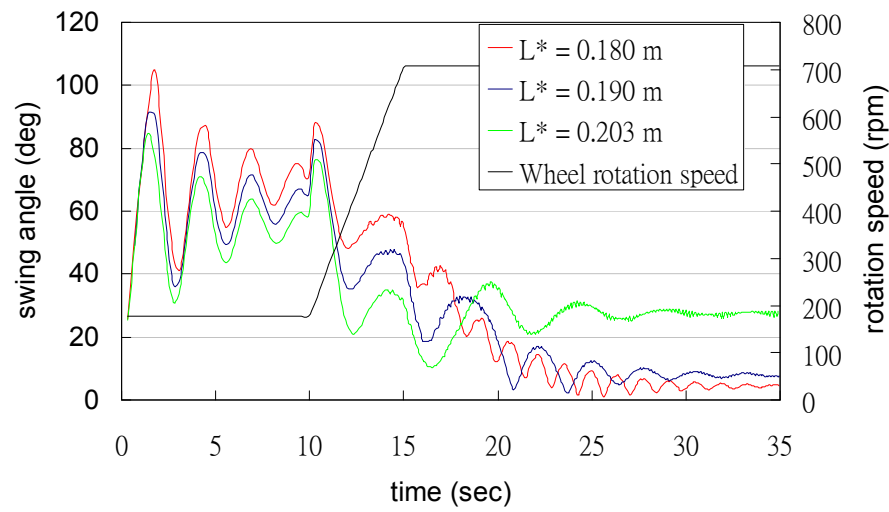

Figure 16. Maximum swing angle under various $L^{*}$ after angular acceleration of $148 \mathrm{rad} / \mathrm{s}^{2}$.

\section{Magnetic circuit and power generation analysis}

Electromagnetic generation is caused by a coil with a dynamically changing magnetic flux density $\mathrm{B}$. To achieve greater power generation at the same oscillation speed with the same number of turns of the coil, a magnetic circuit design for a dense magnetic flux is necessary. Fig. 17 (a) illustrates a novel arrangement of permanent magnets designed using the Halbach array concept. This arrangement consists of 16 individual sector-shaped permanent $\mathrm{Nd}-\mathrm{Fe}-\mathrm{B}$ magnets with an outer radius rout of $13.0 \mathrm{~mm}$, an inner radius rin of $7.0 \mathrm{~mm}$, and a thickness $t_{m}$ of $3.0 \mathrm{~mm}$. The red arrows and symbols represent the flux orientation of each magnet. This circular Halbach array augments the magnetic strength on the coil side of the array. Because of the oscillation motion of the weighted pendulum, a disk-type magnet with a periodic arrangement is considered. 


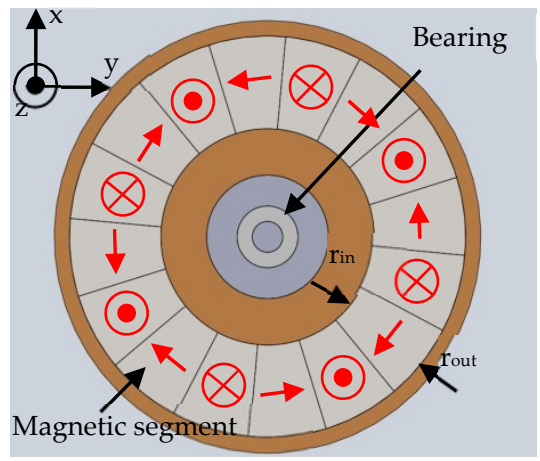

(a)

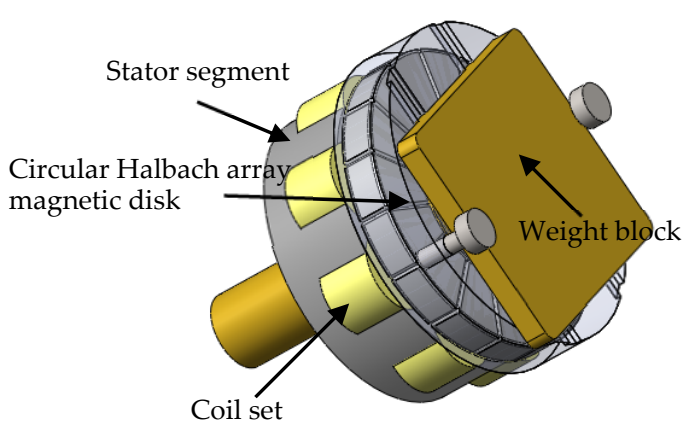

(b)

Figure 17. Schematic illustrations of circular Halbach array magnetic disk.

Fig. 18 presents the 3D magnetic field distribution of the circular Halbach array disk in the A-A cross section plane located between $r_{i n}$ and rout calculated using COMSOL V3.5 software. One period consists of a four-magnet arrangement, and the circular Halbach array disk has four periods. The residual magnetism of each magnet is $1.4 \mathrm{~T}$. The simulation results shown in Fig. 18 indicate that the magnetic flux densities reinforce one another above the array where the coils are placed, but cancel one another below it. This kind of magnetic field distribution increases the induced electromotive force (EMF) $\varepsilon$. Considering the coil motion in the $x-y$ plane, the $z$ component of the magnetic flux density $B_{z}$ above the circular Halbach array was calculated. The gradient change and the strength of the $\mathrm{z}$ component of the average magnetic flux density for the circular Halbach array disk exceed those of normal multipolar magnetic disk. For detailed information on the magnetic circuit analysis, please refer to (Wang et al., 2012b).

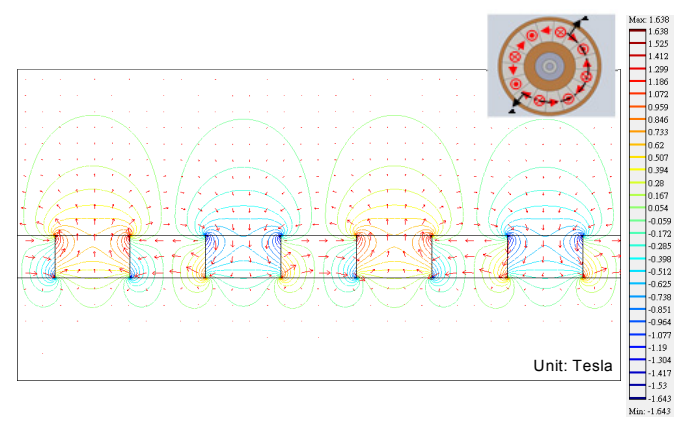

Figure 18. Magnetic field distribution of the circular Halbach array disk.

According to Faraday's law, when a magnet and a coil loop have a relative motion, the changing flux inside the loop can generate an EMF. Considering a coil set in a hollow cylinder shape by spiral winding, the coil set can be divided into $\mathrm{N}_{t}$ layers along the axial direction, and each layer has $\mathrm{N}_{\mathrm{r}}$ loops. The magnetic flux through each coil loop moving in 
the $\mathrm{x}-\mathrm{y}$ plane can be expressed as $u D\left[B_{z}\left(x_{c}+\frac{D}{2}\right)-B_{z}\left(x_{c}-\frac{D}{2}\right)\right]$, where $\mathrm{D}, \mathrm{u}, \mathrm{x}_{\mathrm{c}}$, and $\mathrm{B}_{\mathrm{z}}$ are the diameter of the loop, linear velocity of the center of the loop, center position of the loop, and $\mathrm{z}$ component of magnetic flux density, respectively. The $\mathrm{z}$ component of magnetic flux density of each loop in the different layers is acquired from the simulation results. The total EMF $\varepsilon$ total can be obtained by superposing $\Phi_{i j}(\theta)$ of each coil for the different layers:

$$
\begin{aligned}
& \Phi_{i j}(\theta)=u D_{i j}\left[B_{z i}\left(x_{c_{i j}}+\frac{D_{i j}}{2}\right)-B_{z i}\left(x_{c_{i j}}-\frac{D_{i j}}{2}\right)\right] \\
& \varepsilon_{\text {total }}=\frac{d \Phi_{\text {total }}}{d t}=\frac{d\left(n \sum_{i=1}^{N t} \sum_{j=1}^{N r} \Phi_{i j}(\theta)\right)}{d t}
\end{aligned}
$$

where $n$ is the total number of coil sets. The numerical EMF can be obtained according to the magnetic flux density from above simulation and Eq. (21). The numerical EMF of the circular Halbach array disk was approximately $4.162 \mathrm{~V}$. Applying the chain rule to Faraday's law, the EMF can be expressed as

$$
\varepsilon=\frac{d \Phi}{d t}=\frac{d \Phi}{d \theta} \theta
$$

With EMF and the angular velocity $\dot{\theta}$ acquired from the numerical results, $\mathrm{d} \Phi / \mathrm{d} \theta$ can be calculated by Eq. (22). The torque $M_{m}$ caused by the electromagnetic damping to the rotating system can be represented as

$$
M_{m}(t)=\frac{(d \Phi / d \theta)^{2}}{R_{c}+R_{L}} \dot{\theta}
$$

where $R_{c}$ is the resistance of the coil and $R_{L}$ is the external resistance. Consequently, the electromagnetic damping $\mathrm{C}_{\mathrm{e}}$ can be written as

$$
C_{e}=\frac{(d \Phi / d \theta)^{2}}{R_{c}+R_{L}}
$$

The voltage across the resistor was measured using an oscilloscope, and the power consumed by the external resistor was calculated as the square of the root mean square of the voltage $\left(\mathrm{V}_{\mathrm{rms}}\right)$ divided by the external resistance value (i.e., $\left.P_{\exp }=\left(\mathrm{V}_{\mathrm{rms}}\right)^{2} / \mathrm{R}_{\mathrm{L}}\right)$. Using the electromagnetic damping $\mathrm{C}^{*}$ obtained from Eq. (24) and integrating into the following equation, the numerical power consumption of the external resistor $\left(P_{\text {num. }}\right)$ was calculated as

$$
P_{\text {num. }}=\frac{R_{L}}{\tau\left(R_{c}+R_{L}\right)} \int_{t_{0}}^{t_{0}+\tau} C_{e}^{*} \dot{\theta}^{2} d t
$$


where $\tau$ is the swing period of the weighted pendulum and to is any given time at a steady state. For the proposed design, $\mathrm{d} \Phi / \mathrm{d} \theta$ was approximately $0.066 \mathrm{~V}-\mathrm{s}$, as calculated by Eq. (22). Table 2 lists the magnet and coil parameters of the circular Halbach array disk and Fig. 19 shows the prototype of the energy harvester integrating the weighted pendulum and the circular Halbach array disk. Eqs. (19), (22), and (25) yield the numerical results for power generation and voltage output. Fig. 20 presents the experimental and numerical results for power of the energy harvester (Wang et al., 2012b). The experimental results were averaged from ten sets of data.

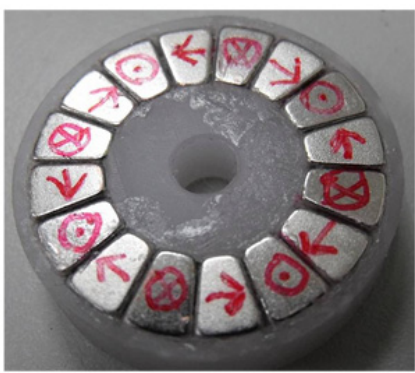

(a)

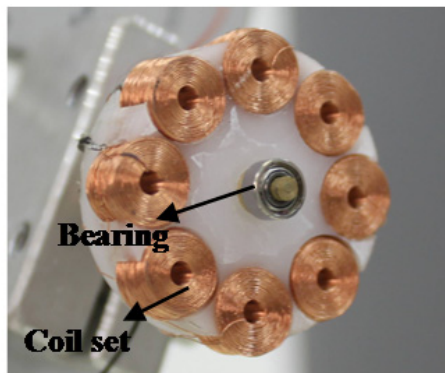

(b)

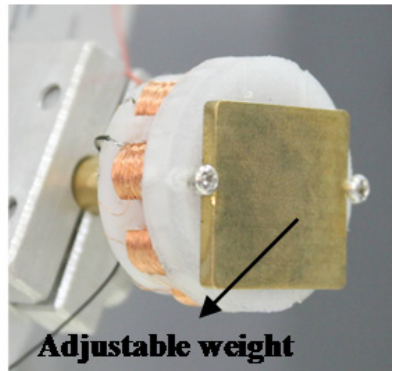

(c)

Figure 19. Prototype of the energy harvester. (a) Rear view of the rotor with the circular Halbach array magnetic disk. (b) Front view of the stator. (c) The energy harvester mounted on the rotation plate.

\begin{tabular}{|l|l|}
\hline Items & Quantification \\
\hline Dimensions of individual magnet & $r_{\text {out }}=13.0 \mathrm{~mm}, r_{\text {in }}=7.0 \mathrm{~mm}, t_{m}=3.0 \mathrm{~mm}$ \\
\hline $\begin{array}{l}\text { Residual magnetism of magnet by axial } \\
\text { magnetization }\end{array}$ & $B_{r}=1.4 \mathrm{~T}$ \\
\hline Coil wire diameter & $d=0.1 \mathrm{~mm}$ \\
\hline Coil turns in a layer and coil layers in a set & $N_{r}=20, \mathrm{~N}_{t}=30$ \\
\hline Thickness of coil set & $t_{c}=3.0 \mathrm{~mm}$ \\
\hline Inside and outside diameter of the coil set & $D_{\text {in }}=2.0 \mathrm{~mm}, D_{\text {out }}=6.0 \mathrm{~mm}$ \\
\hline Air gap between magnetic disk and coil sets & $t_{p}=1.0 \mathrm{~mm}$ \\
\hline Mass Moment of inertia of the rotor & $I=1.611 \times 10^{-6} \mathrm{~kg}-\mathrm{m}^{2}$ \\
\hline $\mathrm{d} \Phi / \mathrm{d} \theta$ & $0.066 \mathrm{~V}-\mathrm{s}$ \\
\hline Coil resistance & $R_{c}=156 \Omega$ \\
\hline
\end{tabular}

Table 2. Parameters of the circular Halbach array disk for the power generation experiments.

Numerical and experimental results both demonstrate that the electrical power obtained from the weighted pendulum increased monotonically with wheel speed because of the high angular velocity $\theta$ of the magnets, which induced a large EMF $\varepsilon$. The difference between the numerical and experimental results in terms of the voltage across the resistor was smaller than that observed in the average power output because power output contains the squared term of voltage. In the power output experiments, the optimum external 
resistance required to obtain the largest power output ranged from $330 \Omega$ to $510 \Omega$, which is close to the internal resistance of the coil, but not constant. These results confirm that maximum power is delivered to an external load when the external load resistance equals the sum of the coil resistance and the electrical analogue of the friction damping coefficient. Although a high external resistance can produce a large output voltage for greater efficiency in the pumping circuit, a large power output occurs at an optimum resistance. Hence, there must be a trade-off between circuit design and power transformation.

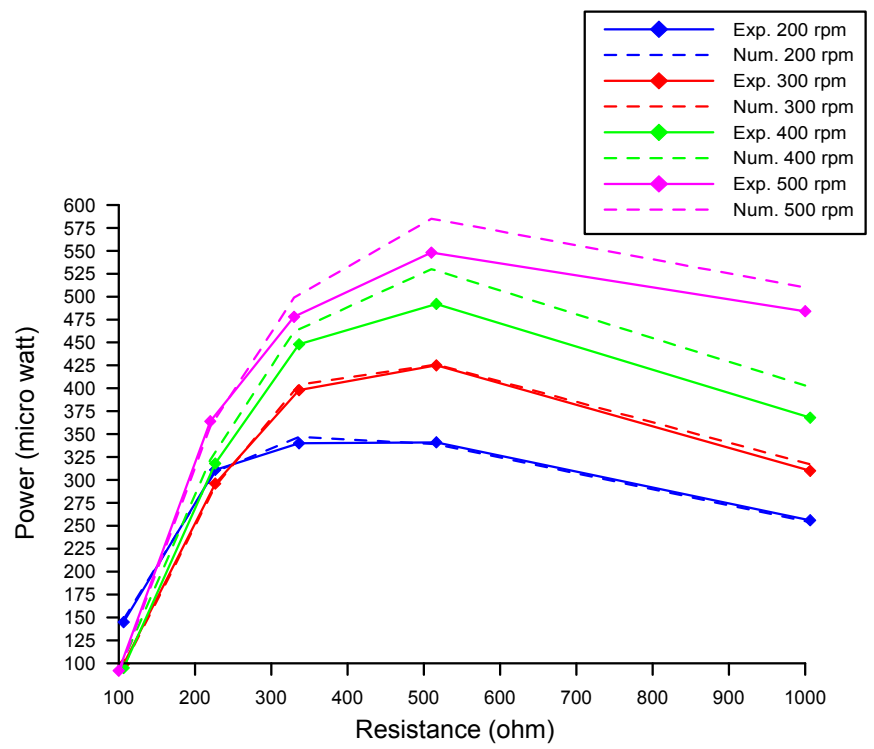

Figure 20. Experimental and numerical results for the circular Halbach array disk. Average power output (consumption) of the external resistor (unit, $\mu \mathrm{W}$ ).

\section{Conclusion}

This chapter proposes a novel well-weighted pendulum design. Numerical analysis and experimental results reveal that several hundred micro-Watts of power, which is sufficient to drive a TPMS, can be harvested from this well-weighted pendulum. If the characteristic length $L^{*}$ is equal to $R_{2}$, then the natural frequency of oscillation of the well-weighted pendulum matches the rotational frequency of wheel at any wheel speed. Consequently, resonance will occur at any wheel speed. Therefore, oscillation at a large angle and velocity for large power generation is feasible.

The magnetic field simulation results in this study show that the novel circular Halbach array disk exhibits a high gradient change and large strength of the $\mathrm{z}$ component of the average magnetic flux density because it reinforces the magnetic flux density inside the area in which the coils are placed. This in turn leads to a large power generation. The simulation models of the power generation were built well on the basis of the 3D magnetic field distribution by COMSOL V3.5, the numerical EMF equations, and Faraday's law. 
The analytical model of power generation employed for numerical simulation, which showed good agreement with the experimental results, was developed based on models presented in this study. At an optimum external resistance, the power output of the energy harvester integrating the well-weighted pendulum and the multipolar magnetic disk was approximately 300-550 micro-Watts at $200-500 \mathrm{rpm}$. These results demonstrate that this has the potential to replace a TPMS battery.

\section{Author details}

Yu-Jen Wang, Sheng-Chih Shen and Chung-De Chen

National Formosa University, National Cheng Kung University, Industrial Technology Research Institute, Taiwan

\section{Acknowledgement}

The authors would like to thank National Science Council (NSC), and College of Engineering and Research Center for Energy Technology and strategy, National Cheng Kung University for their financial supports to the project (granted number: NSC 98-2221-E006-260-MY3 and 100-2628-E-006-019-MY3). Moreover, the authors wish to thank SouthIndustrial Technology Research Institute (S-ITRI) and National Formosa University (NFU) for their related supports.

\section{References}

Bowers, B. J. \& Arnold, D. P. (2009). Spherical, rolling magnet generators for passive energy harvesting from human motion, J. Micromech. Microeng., Vol. 19, No. 9, 2009, 094008, ISSN 0960-1317.

Dallago, E.; Marchesi, M. \& Venchi, G. (2010). Analytical model of a vibrating electromagnetic harvester considering nonlinear effects, IEEE Transactions on Power Electronics, Vol. 25, No. 8, Aug. 2010, pp. 1989-1997, ISSN 0885-8993.

Daminakis, M.; Goethals, J. \& Kowtke, J. (2005). Enhancing Power Harvesting using a Tuned Auxiliary Structure, Journal of Intelligent Material Systems and Structures, Vol. 16, No. 10, (2005) 825-834, ISSN 1045-389X.

$\mathrm{Gu}, \mathrm{L}$. \& Livermore, C. (2010). Passive self-tuning energy harvester for extracting energy from rotational motion, Applied Physics Letters, 97, 2010, 081904, ISSN 0003-6951.

Hatipoglu, G. \& Urey, H. (2009). FR4-based electromagnetic energy harvester for wireless tire sensor nodes, Proceedings of the Eurosensors XXIII conference, Procedia Chemistry 1, 2009, pp. 1211-1214, ISSN 1876-6196.

Lallart, M.; Anton, S. R. \& Inman, D. J. (2010). Frequency Self-tuning Scheme for Broadband Vibration Energy Harvesting, Journal of Intelligent Material Systems and Structures, Vol. 21, No. 9, 2010, pp. 897-906, ISSN 1045-389X.

Lee, D.G.; Carman, G.P.; Murphy, D. \& Schulenburg, C. (June 2007). Novel micro vibration energy harvesting device using frequency up conversion, TRANSDUCER \& 
EUROSENSORS '07, The 14th International Conference on Solid-state Sensors, Actuators and Microsystems, 10-14.

Leland, E.S. \& Wright, P.K. (2006). Resonance tuning of piezoelectric vibration energy scavenging generators using compressive axial preload, Smart Mater. Struct., 15 (2006) 1413-1420, ISSN 0964-1726.

Lohndorf, M.; Kvisterøy, T.; Westby, E. \& Halvorsen, E. (2007). Evaluation of energy harvesting concepts for tire pressure monitoring systems, Technical Digest PowerMEMS 2007, Freiburg, Germany, (2007) 331-334.

Mansour, M. O.; Arafa, M. H. \& Megahed, M. (2010). Resonator with magnetically adjustable natural frequency for vibration energy harvesting, Sensors and Actuators A, 163, 2010, pp. 297-303, ISSN 0924-4247.

Marinkovic, B. \& Koser, H. (2009). Smart sand - a wide bandwidth vibration energy harvesting platform, Applied Physics Letters, 94, 103505, 2009, ISSN 0003-6951.

Marzencki, M.; Defosseux, M. \& Basrour, S. (2009). MEMS Vibration Energy Harvesting Devices With Passive Resonance Frequency Adaptation Capability, Journal of Microelectromechanical Systems, Vol. 18, No. 6, 2009, pp. 1444-1453, ISSN 1057-7157.

Matsuzaki, R. \& Todoroki, A. (2008). Wireless monitoring of automobile tires for intelligent tires, Sensors, 8 (2008) 8123-8138, ISSN 1424-8220.

Platt, S. R.; Farritor, S. \& Haider, H. (2005). On low-frequency electric power generation with PZT ceramics, IEEE/ASME Transactions on Mechatronics, Vol. 10, No. 2, April 2005, pp. 240-252, ISSN 1083-4435.

Roundy, S. (2008). Energy harvesting for tire pressure monitoring systems: design considerations, Technical Digest PowerMEMS 2008, Sendai, Japan, (2008) 1-6.

Sari, I.; Balkan, T. \& Kulah, H. (2008). An electromagnetic micro power generator for wideband environmental vibrations, Sensors and Actuators A., 145-146, 405-413, ISSN 0924-4247.

Shahruz, S.M. (2006). Design of mechanical band-pass filters for energy scavenging, Journal of Sound and Vibration, 292 (2006) 987-998, ISSN 0022-460X.

Stanton, S.; McGehee, C. \& Mann, B. (2009). Reversible hysteresis for broadband magnetopiezoelastic energy harvesting, Applied Physics Letters, 95, 174103, ISSN 0003-6951.

Wang, Y. J.; Chen, C. D. \& Sung, C. K. (2012). System design of a weighted-pendulum type electromagnetic generator for harvesting energy from a rotating wheel, IEEE/ASME Transactions on Mechatronics, ID: 10.1109/TMECH.2012.2183640, Vol. 99, 2012, pp. 1-10, ISSN 1083-4435.

Wang, Y. J.; Chen, C. D.; Sung, C. K. \& Li, C. (2012). Natural Frequency Self-tuning Energy Harvester using a Circular Halbach Array Magnetic Disk, Journal of Intelligent Material Systems and Structures, 23, 8, 933-943, ISSN 1045-389X.

Youngsman, J. M.; Luedeman, T.; Morris, D. J.; Anderson, M. J. \& Bahr, D. F. (2010). A model for an extensional mode resonator used as a frequency-adjustable vibration energy harvester, Journal of Sound and Vibration, 329, 2010, pp. 277-288, ISSN 0022-460X.

Zhang, C. L. \& Chen, W. Q. (2010). A wideband magnetic energy harvester, Applied Physics Letters, 96, 123507, 2010, ISSN 0003-6951. 